\title{
Adsorption of Alkyltriphenylphosphonium Amphiphiles on Nafion Membranes. X-ray Photoelectron Spectroscopy and Static Secondary Ion Mass Spectrometry Analysis
}

\author{
Wilma F. van Straaten-Nijenhuis, Ernst J. R. Sudhölter, ${ }^{\dagger}$ Feike de Jong, and \\ David N. Reinhoudt ${ }^{*}$ \\ Laboratory of Organic Chemistry, University of Twente, P.O. Box 217, \\ 7500 AE Enschede, The Netherlands \\ Jan W. G. Mahy \\ Akzo Research Laboratories, Corporate Research, P.O. Box 9300, \\ 6800 SB Arnhem, The Netherlands
}

Received October 10, 1992. In Final Form: April 27, 1993

\begin{abstract}
Conductivity, UV, and attenuated total reflectance IR measurements show that $n$-alkyltriphenylphosphonium amphiphiles adsorb on a Nafion 117 membrane. Approximately $20 \%$ of the Nafion protons are exchanged for a cationic amphiphile ( $n$-hexadecyltriphenylphosphonium). Diffusion of amphiphile through the membrane was not observed. Once adsorbed, the amphiphiles did not leach from the membrane. Surface-sensitive techniques ( $x$-ray photoelectrion spectroscopy, static secondary ion mass spectrometry) were used to investigate the presence, concentration, and distribution of the amphiphile in the Nafion membrane. Our experiments point to an incomplete monolayer coverage of the membrane, the molar ratio of amphiphile to sulfonate groups being only slightly less than 1 in the uppermost 2-5 nm. The amphiphile is bonded to the membrane, most likely via an ionic bond with the sulfonate groups. X-ray fluorescence measurements show that the amphiphile is also present in the bulk of the membrane, at least in the uppermost micrometer. However, in the bulk the concentration of amphiphile is significantly lower than the sulfonate groups. These results show that thin and stable amphiphilic layers can be made on a solid support material using adsorption of an amphiphile and coupling via an ionic bond.
\end{abstract}

\section{Introduction}

In the past decade there has been an increasing interest in the formation of thin amphiphilic layers on solid support materials. ${ }^{1}$ An established technique for the formation of mono- or multilayers is the Langmuir-Blodgett technology, by which monolayers at the air/water interface are organized and subsequently transferred to a solid substrate by (repeated) dipping of the substrate through the interface. $^{2-4}$ This technique requires special precautions with respect to the purity of the chemicals used and the room where the experiments are performed and is limited to amphiphiles that form stable monolayers at the air/ water interface. Defects and voids can be easily introduced on transferring the monolayer, and the stability of the layers is often low.

An alternative method for the organization of monolayers on a solid support is the adsorption and self-assembly of amphiphiles. Bigelow and Zisman have reported the formation of monolayers on polar substrates such as glass and different metals by adsorption from an organic solution..$^{5,6}$ Sagiv $^{7,8}$ and others ${ }^{9-11}$ have used derivatives

${ }^{\dagger}$ Present address: Wageningen Agricultural University, Laboratory of Organic Chemistry, Dreijenplein 8, 6703 HB Wageningen, The Netherlands. 1990 .

(1) Roberts, G. Langmuir-Blodgett Films; Plenum Press: New York,

(2) Blodgett, K. A.; Langmuir, I. Phys. Rev. 1937, 51, 964.

(3) Tieke, B. Adv. Mater. 1991, 3, 532.

(4) Ringsdorf, H.; Schlarb, B.; Venzmer, J. Angew. Chem., Int. Ed. Engl. 1988, 27, 113

(5) Bigelow, W. C.; Pickett, D. L.; Zisman, W. A. J. Colloid Sci. 1946, 1,513 .

(6) Bigelow, W. C.; Brockway, L. O. J. Colloid Sci. 1956, 11, 60.

(7) Sagiv, J. J. Am. Chem. Soc. 1980, 102, 92

(8) Maoz, R.; Sagiv, J. Langmuir 1987, 3, 1034.

(9) Wasserman, S. R.; Tao, Y.-T.; Whitesides, G. M. Langmuir 1989, 5,1074 . of alkyltrichlorosilanes for the adsorption on polar surfaces followed by chemical reaction. Multilayers have been made from monolayers containing terminal functional groups that can be deprotected for the adsorption and chemical binding of the next layer.11,12 Other methods include the self-assembly of alkanethiols on gold, silver, and copper surfaces, ${ }^{13,14}$ of dialkyl sulfides ${ }^{15,16}$ and dialkyl disulfides ${ }^{17,18}$ on gold, of fatty acids on alumina, ${ }^{19-21}$ and of alcohols and amines on platinum. ${ }^{5}$ In all these cases there are strong molecule-substrate interactions. The layers formed by adsorption are highly ordered and usually more stable than LB films.

We are interested in the selective membrane transport of salts assisted by synthetic macrocyclic receptors through supported liquid membranes. ${ }^{22-24}$ In these systems the transport is limited by the diffusion of the complex and

(10) Kessel, C. R.; Granick, S. Langmuir 1991, 7, 532.

(11) Ulman, A. Adv. Mater. 1990, 2, 573.

(12) Netzer, L.; Sagiv, J. J. Am. Chem. Soc. 1983, 105, 674

(13) Laibinis, P. E.; Whitesides, G. M.; Allara, D. L.; Tao, Y.-T.; Parikh, A. N.; Nuzzo, R. G. J. Am. Chem. Soc. 1991, $113,7162$.

(14) Diem, T.; Czajka, B.; Weber, B.; Regen, S. L. J. Am. Chem. Soc. 1986, 108,6094

(15) Throughton, E. B.; Bain, C. D.; Whitesides, G. M.; Nuzzo, R. G.; Allara, D. L.; Porter, M. D. Langmuir 1988, 4, 365.

(16) Li, T. T.-T.; Weaver, M. J. J. Am. Chem. Soc. 1984, 106, 6107.

(17) Nuzzo, R. G.; Fusco, R. A.; Allara, D. L. J. Am. Chem. Soc. 1987, 109,2358 .

(18) Taniguchi, I.; Toyosawa, K.; Yamaguchi, H.; Yasukouchi, K. $J$. Chem. Soc., Chem. Commun. 1982, 1032.

(19) Chen, S. H.; Frank, C. W. Langmuir 1989, 5, 978.

(20) Allara, D. L. Langmuir 1985, 1,45 and 62.

(21) Ogawa, H.; Chihara, T.; Taya, K. J. Am. Chem. Soc. 1985, 107, 1365.

(22) Stolwijk, T. B.; Sudhölter, E. J. R.; Reinhoudt, D. N. J. Am. Chem. Soc. $1987,109,7042$.

(23) Nijenhuis, W. F.; Buitenhuis, E. G.; de Jong, F.; Sudhơlter, E. J.

R.; Reinhoudt, D. N. J. Am. Chem. Soc. 1991, 113, 7963.

(24) Nijenhuis, W. F.; Walhof, J. J. B.; Sudhölter, E. J. R.; Reinhoudt, D. N. Recl. Trav. Chim. Pays Bas 1991, 110, 265. 


$$
-\left[\left(\mathrm{CF}_{2}-\mathrm{CF}_{2}\right)_{\mathrm{k}}-\left(\mathrm{CF}_{2}-\mathrm{CF}\right)_{1}\right]_{\mathrm{n}^{-}}-\mathrm{O}-\left(\mathrm{CF}_{2}-\mathrm{CF}-\mathrm{O}\right)_{\mathrm{m}}-\left(\mathrm{CF}_{2}\right)_{2}-\mathrm{SO}_{3} \mathrm{H}
$$

$k=6.5,1=1, m=1, n=c a .1000$

Figure 1. Chemical structure of Nafion 117.

is therefore inversely proportional to the thickness of the membrane. The thinnest membranes consist of monolayers of amphiphiles but these layers are often not mechanically stable. This problem can be overcome by using a monolayer on a support. Obviously, the latter has to be highly salt-permeable, a requirement that precludes the use of the substrates described above (metals and oxides). Therefore, we decided to study the adsorption of amphiphiles on Nafion, a perfluorinated polymer containing sulfonic acid groups. ${ }^{25}$ The generalized structure of Nafion 117 is given in Figure 1. Nafion is an extremely stable material containing hydrophobic ( $\mathrm{CF}_{2}$ segments) as well as hydrophilic domains $\left(\mathrm{SO}_{3} \mathrm{H}\right.$ channels) and is commonly used as a cation-exchanger. ${ }^{26}$ In a polar medium the concentration of sulfonic acid groups at the surface is high and we envisaged the use of these groups for the adsorption of cationic amphiphiles via an ion exchange process.

Firstly, we have studied the adsorption of commercially available cationic amphiphiles. The adsorption process was monitored by conductometry and UV spectroscopy and the modified membranes were analyzed by attenuated total reflection infrared spectroscopy (ATR-IR), X-ray photoelectron spectroscopy (XPS), and static time-offlight secondary ion mass spectrometry (static ToFSIMS). ${ }^{27}$ XPS probes in a quasi-nondestructive way the uppermost 5-10 $\mathrm{nm}$ of the sample by detection of the core level photoelectron kinetic energy, excited by X-rays such as $\mathrm{Mg} \mathrm{K} \alpha$ or $\mathrm{Al} \mathrm{K} \alpha$; $^{26}$ from the knowledge of incident $\mathrm{X}$-ray energy and kinetic photoelectron energy, the core level binding energy, which is characteristic of each element, can be calculated (taking into account the instrument work function). ${ }^{27 a}$ By systematically decreasing the detection angle relative to the sample surface, the information-depth in the sample can be decreased (angleresolved XPS, ARXPS).

XPS yields semiquantitative information on the surface concentration of elements as well as element binding states, averaged over the analyzed surface area. From these data and using the appropriate surface structure model, the amount of amphiphile present in the surface region can be calculated. The distinction of different binding sites of the elements allows furthermore to obtain information on the concentration of functional groups at the surface.

With static SIMS the mass of the (positive or negative) secondary ion fragments emitted from the uppermost 1 $\mathrm{nm}$ is detected. 27b Under static conditions, the high-energy (30 kV) low-current (typically below $1 \mathrm{pA}$ ) primary ion beam probes the surface in such a way that (by definition) during the experiment less than $1 \%$ of a monolayer of the surface is sputtered, i.e. quasi-nondestructive analysis also.

Static SIMS imaging makes use of the finely focused scanning primary ion source, as well as of the time-of-

(25) Sondheimer, S. J.; Bunce, N. J.; Fyfe, C. A. J. Macromol. Sci. Rev. Macromol. Chem. Phys. 1986, C26, 353.

(26) Stinson, S. C. Chem. Eng. News 1982, March 15, 22.

(27) (a) Practical Surface Analysis, 2nd ed.; Briggs, D., Seah, M. P. Eds.; J. Wiley \& Sons: Chichester, UK, 1990; Vol. 1. (b) Benninghoven, A.; Rüdenauer, F. G.; Werner, H. W. Secondary Ion Mass Spectrometry J. Wiley \& Sons: Chichester, UK, 1987. (c) Stickle, W. F.; Moulder, J. F. J. Vac. Sci. Technol. A 1991, 9 (3), 1441. flight (ToF) detection mode to obtain images of the lateral (surface) distribution of the secondary ions (elements, fragments). The lateral resolution is of the order of $1 \mu \mathrm{m}$, which may provide information on the distribution of the amphiphile (e.g. in domains) at the surface.

The combination of these complementary surface sensitive techniques allows information to be obtained of the surface element concentrations (XPS) as well as of the microscopical distribution of moieties at the surface (imaging SIMS) in the range of a few to around $10 \mathrm{~nm}$ (ARXPS), as well as in the uppermost $1 \mathrm{~nm}$. The combination of local (binding state, XPS) chemistry and long-range (fragments, Static SIMS) chemistry may provide a complete picture of the polymer surface. ${ }^{27 \mathrm{c}}$

Of course, it should be emphasized that this combined information refers to the structure of the dry surface (in vacuum) and not to the structure in contact with the aqueous solution from which it is obtained.

\section{Experimental Section}

Materials. Nafion 117 (equivalent weight $1100,0.018 \mathrm{~cm}$ thick), a registered trademark of DuPont, was obtained from Aldrich. The membranes were cut into pieces with a diameter of $4.5 \mathrm{~cm}$. The pieces were boiled in bidistilled, deionized water for $4 \mathrm{~h}$, after which they were soaked in $1 \mathrm{~N} \mathrm{HCl}$ for $24 \mathrm{~h}$. The membranes were rinsed with water and the surface layer of water was carefully removed by dipping with a tissue before measuring. The membranes were stored in bidistilled, deionized water. Teflon was obtained from DuPont. The amphiphiles are all commercially available: $n$-octyl-, $n$-decyl-, and $n$-hexadecyltriphenylphosphonium bromide (Alfa Products), $n$-tetradecyltrimethylammonium bromide, $n$-hexadecyltrimethylammonium bromide, and $n$-hexadecylpyridinium chloride (Janseen Chimica), $n$-dodecylpyridinium chloride and $n$-didecyldimethylammonium bromide (Aldrich), $n$-decyltrimethylammonium bromide (Eastman Kodak), and $n$-didodecyldimethylammonium bromide (Fluka). All amphiphiles were of reagent grade and were used without further purification. In all cases bidistilled and deionized water was used.

Adsorption Measurements. The Nafion membrane was placed in a permeation cell consisting of two identical cylindrical compartments (half-cell volume, $50 \mathrm{~mL}$; effective membrane area $12.4 \mathrm{~cm}^{2}$ ) that has been described previously. ${ }^{22}$ Each compartment was stirred at a speed of $1000 \mathrm{rpm}$. The measurements were performed at a constant temperature of $25^{\circ} \mathrm{C}$ and at least in duplicate. Only for these measurements to the aqueous solutions of $25 \mathrm{mM}$ amphiphile $0.1 \mathrm{M} \mathrm{KCl}$ was added as electrolyte, and these solutions were placed on each side of the membrane. ${ }^{28}$ The adsorption was monitored by measuring the conductivity of the aqueous phases as well as the conductivity over the membrane (using an electrode on each side of the membrane) as a function of time (Philips PW 9527 conductivity meter and Philips PW 9512/61 electrodes with cell constants of $0.76 \mathrm{~cm}^{-1}$ ). When the conductivity over the membrane was measured, the cell was calibrated and the cell constant was determined experimentally by using standard $\mathrm{KCl}$ solutions with known conductivity. In the case of $n$-hexadecyltriphenylphosphonium bromide (HTPB), the initial amphiphile concentration was varied (no electrolyte used) to determine the adsorption isotherm. Samples of the aqueous phase were taken after $24 \mathrm{~h}$ and the UV absorption at $268 \mathrm{~nm}$ was measured. The concentration of amphiphile was calculated from this absorption using a calibration curve. Leaching of adsorbed amphiphile from the membrane was verified by replacing the amphiphile solutions several times with water. After $24 \mathrm{~h}$ the concentration of amphiphile in the aqueous phases was determined by UV spectroscopy. To verify the diffusion of the amphiphile through the membrane, two membranes clamped together were placed between the two half-cells and a 25 mM solution of HTPB was

(28) Only in the case of the conductivity measurements $0.1 \mathrm{M} \mathrm{KCl}$ was added to the amphiphile solution to avoid the conductivity over the membrane being too low. In all other measurements no electrolyte was
used. 
placed on one side of the membranes. The receiving aqueous phase was analyzed by UV spectroscopy after $24 \mathrm{~h}$ and both membranes were analyzed by ATR-IR spectroscopy (vide infra).

Analysis of the Modified Nafion. Before analysis, the modified membranes ${ }^{28}$ were thoroughly rinsed with bidistilled and deionized water. ATR-IR spectra were recorded on a Nicolet 5 SXC FT-IR spectrometer, using a germanium crystal ( $50 \times 20$ $\times 2 \mathrm{~mm}$ ) and an angle of incidence of $45^{\circ}$. The XPS spectra were obtained using a VG Escalab MkII spectrometer. The sample surface was irradiated with non-monochromatized $\mathrm{Mg} \mathrm{K} \alpha \mathrm{X}$-rays. The emitted core electrons were detected as a function of their kinetic energy, using the "constant analyzer energy" mode. For quantification purposes, the pass energy was set at $50 \mathrm{eV}$, while for curve fitting a better energy resolution was obtained at $20 \mathrm{eV}$ pass energy. Data acquisition was performed using the VGS 5000 software package and data processing using the VGS 5250 enhanced data package. The Wagner set ${ }^{29}$ of empirically derived relative sensitivity factors for the element core levels were used. A Shirley type background correction was applied. ${ }^{30}$ Curve fitting routines involved iterative optimization of position, height, fwhm, and Gauss/Lorentz shape function for a user-defined number of peaks.

The SIMS spectra were recorded using a VG IX23LS timeof-flight instrument. A metal ion gun with liquid $\mathrm{Ga}$ was used a produce a "static" (i.e. $10^{12}$ ions $\mathrm{cm}^{-2}$ per experiment) primary ion source (pulsed $10 \mathrm{kHz}, 30 \mathrm{kV}, 0.01 \mathrm{pA}$ ). The secondary ions generated from the observed sample surface of about $50 \mu \mathrm{m} \times$ $50 \mu \mathrm{m}$ were detected with a Poschenrieder type energy focusing time-of-flight detector. The instrument was described elsewhere. ${ }^{31}$

\section{Results and Discussion}

Adsorption of Amphiphiles on a Nafion Membrane. The adsorption of several commercially available cationic amphiphiles on a Nafion membrane was studied by measuring the condutivity both in the aqueous amphiphile solution and over the membrane. Amphiphiles were studied having different cationic head groups (trimethylammonium, dimethylammonium, pyridinium, and triphenylphosphonium), one or two alkyl chains, and different alkyl chain lengths ( $n$-octyl, $n$-decyl, $n$-dodecyl, $n$-tetradecyl, and $n$-hexadecyl) (see Experimental Section). Aqueous solutions of $25 \mathrm{mM}$ of amphiphile and $0.1 \mathrm{M}$ of $\mathrm{KCl}$ as an electrolyte were used. Of the amphiphiles studied the $n$-alkyltriphenylphosphonium amphiphiles showed the most stable adsorption behavior and these amphiphiles were studied in more detail. The results of the conductivity measurements of the $n$-alkyltriphenylphosphonium bromides are given in Figure 2. Figure 2 shows that the conductivity in the aqueous phases increases slightly and that the conductivity over the membrane decreases dramatically in time for all three $n$-alkyltriphenylphosphonium amphiphiles. Both effects can be explained by the exchange of $\mathrm{H}^{+}$and $\mathrm{K}^{+}$counterions from the $\mathrm{SO}_{3} \mathrm{H}$ groups of Nafion for a cationic amphiphile: because in the aqeuous solution a large and less mobile cation is replaced by a small and more mobile proton, the bulk conductivity increases, and the conductivity over the membrane decreases. Without adsorbed amphiphile, there is a fast cation transport $\left(\mathrm{K}^{+} / \mathrm{H}^{+}\right.$exchange) through the membrane and, therefore, a low electrical resistance over the membrane. When cationic amphiphiles are adsorbed via an ionic bond to the $\mathrm{SO}_{3}$ - groups, there are less protons available for cation exchange and also the pores of the membrane might be blocked by the large amphiphiles. In order to obtain more quantitative information, we have measured the concentrations of

(29) Wagner, C. D. Anal. Chem. 1977, 49, 1282

(30) Shirley, D. A. Phys. Rev. B 1972, 5, 4709

(31) Waugh, A. R.; Kingham, D. R.; Richardson, C. H.; Goff, M. J. Phys., Coll. C6 1987, 48, 577.

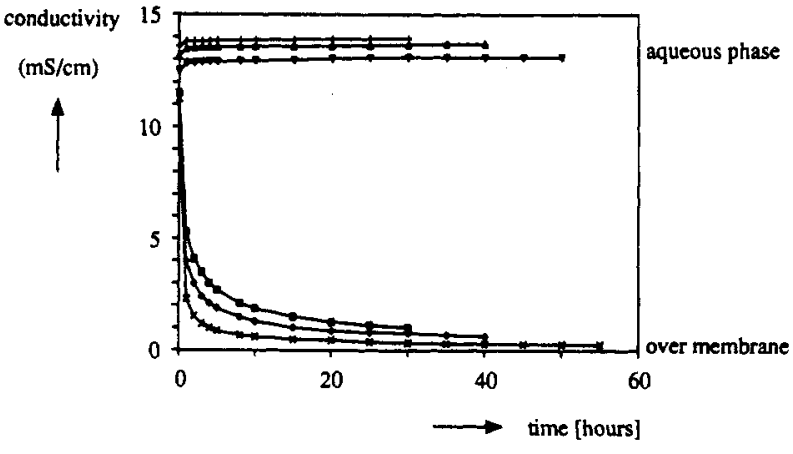

$+=$ bulk; $n$-octyl
$\Delta=$ bulk; $n$-decyl
$\nabla=$ bulk; $n$-hexadecyl
$0=$ over Nafion; $n$-octyl
$\forall=$ over Nafion; $n$-decyl
$x=$ over Nafion; $n$-hexadecyl

Figure 2. Conductivity experiments of the adsorption of $n$-alkyltriphenylphosphonium amphiphiles (aqueous solution of $25 \mathrm{mM}$ amphiphile and $0.1 \mathrm{M} \mathrm{KCl}$ as electrolyte) on a Nafion 117 membrane $\left(T=25^{\circ} \mathrm{C}\right)$.

$n$-hexadecyltriphenylphosphonium bromide (HTPB) in the aqueous phases in the absence of $\mathrm{KCl}$ before and after adsorption by UV spectroscopy at a fired wavelength ( 268 $\mathrm{nm}$ ) using different starting concentrations. At concentrations higher than $4 \mathrm{mM}$ of amphiphile, the amount of amphiphile adsorbed on the Nafion membrane in $24 \mathrm{~h}$ reaches a plateau value of $0.04 \mathrm{mmol}$. From the membrane area $\left(12.4 \mathrm{~cm}^{2}\right)$, the membrane thickness $(0.18 \mathrm{~mm})$, the density $(1.97 \mathrm{~kg} / \mathrm{L})$, and the equivalent weight of the polymer $(1100 \mathrm{~g} /$ equiv) it follows that the Nafion membrane contains $0.4 \mathrm{mmol}$ of $\mathrm{SO}_{3}-$. This means that only $20 \%$ of the Nafion protons is exchanged for an amphiphile.

The presence of amphiphiles on the membrane was confirmed by ATR-IR. Although ATR-IR is known as a surface-sensitive technique, ${ }^{32}$ the penetration depth of the IR light is quite large $(0.1-2 \mu \mathrm{m})$, depending on the type of crystal used, the wavelength, and the angle of incidence. ATR-IR experiments were performed on Nafion membranes modified with $n$-octyl, $n$-decyl-, and $n$-hexadecyltriphenylphosphoniun amphiphiles. In addition to the Nafion signals clear $\mathrm{CH}_{2}$ and $\mathrm{CH}_{3}$ vibrations $\left(2927 \mathrm{~cm}^{-1}\right)$ as well as the P-phenyl vibrations $\left(1442 \mathrm{~cm}^{-1}\right)$ were observed in all cases.

In order to verify whether the amphiphiles adsorb also inside the membrane and/or diffuse through the mem. brane, two Nafion membranes were clamped together and placed in contact with a $25 \mathrm{mM}$ aqueous solution of HTPB in the absence of $\mathrm{KCl}$ on one side of the membrane and water (receiving phase) on the other side. ATR-IR measurements confirmed the presence of amphiphiles on the first membrane, while no amphiphile was detected on the second membrane. These results clearly indicate that ion exchange in deeper regions of the membrane can be excluded.

The stability of the adsorbed layer of amphiphiles was investigated in the following experiment. A solution of 25 mM of HTPB placed on both sides of a Nafion membrane was replaced by water after $24 \mathrm{~h}$ and subsequently the aqueous phase was analyzed by UV spectroscopy and the membrane by ATR-IR measurements. This was repeated several times and the ATR-IR spectra showed in all cases strong HTP absorption. By use of UV spectroscopy, no significant amount of amphiphile could be detected in the

(32) Knutsen, K.; Lyman, D. J. In Surface and Interfacial Aspects of Biomedical Polymers; Andrade, J. D., Ed.; Plenum Press: New York, 1985; Vol. 1: Surface and Chemistry Physics, Chapter 6. 
Table I. Surface Elemental Concentrations Detected by XPS (atom \%)

\begin{tabular}{lcccccc}
\hline \multicolumn{1}{c}{ sample } & $\% \mathrm{C}$ & $\% \mathrm{O}$ & $\% \mathrm{~F}$ & $\% \mathrm{~S}$ & $\% \mathrm{P}$ & $\% \mathrm{Br}$ \\
\hline HTPB & 90.5 & 3.2 & & & 3.3 & 3.0 \\
Nafion & 39.9 & 4.3 & 55.1 & 0.5 & & \\
Nafion corr & 32.6 & 4.8 & 62.0 & 0.6 & & \\
Nafion + HTP & 46.7 & 6.1 & 45.4 & 1.2 & 0.7 & \\
id. 45 & 46.6 & 6.0 & 45.7 & 1.0 & 0.7 & \\
id. $30^{\circ}$ & 44.8 & 5.9 & 47.7 & 1.0 & 0.5 &
\end{tabular}



Table II. Theoretical Element Concentrations for Nafion and HTPB

\begin{tabular}{ccccccc}
\hline sample & $\% \mathrm{C}$ & $\% \mathrm{O}$ & $\% \mathrm{~F}$ & $\% \mathrm{~S}$ & $\% \mathrm{P}$ & $\% \mathrm{Br}$ \\
\hline HTPB & 94.4 & & & & 2.8 & 2.8 \\
Nafion & 30.8 & 7.7 & 60.0 & 1.5 & &
\end{tabular}

aqueous phases on consecutive replacements. This means that the amphiphiles are chemisorbed by ion-exchange.

XPS and SIMS Analysis of Modified Nafion. Additional information on the adsorbed amphiphiles was obtained from XPS and static ToFSIMS surface analysis of the membranes. As obtained Nafion, HTP modified Nafion, and as obtained HTPB have been analyzed. The XPS results are summarized in Table $I$.

The bulk elemental compositions for pure Nafion $\left(\mathrm{C}_{20} \mathrm{~F}_{39} \mathrm{O}_{5} \mathrm{~S}\right)^{33,35}$ and the pure amphiphile $\left(\mathrm{C}_{34} \mathrm{PBr}\right)$ obtained from the literature are given in Table II. In order to be compared with the experimental data, the relative concentrations do not include hydrogen, since this element is not detected by XPS.

Comparison of Tables I and II shows good agreement between the bulk and the surface elemental compositions. The observed higher surface $\mathrm{C} / \mathrm{F}$ ratio can be attributed to irradiation induced surface degradation of the membrane. The strong X-ray induced surface degradation of halogenated polymer systems is well-known from the literature (see e.g. Briggs in ref 27a). Since this phenomenon was expected, particularly while using a nonmonochromatized X-ray source, the analysis time was purposedly limited at the expense of the signal-to-noise ratio in order to minimize this degradation. The degradation manifests itself in the XPS spectrum in both the $C(1 s)$ and $F(1 s)$ signals as an irradiation-time-dependent growth of the contributions associated with carbon atoms bonded only to carbon and hydrogen, or of fluoride $\left(\mathrm{F}^{2}\right)$, respectively. It therefore can be taken into account, if the total carbon and fluorine concentration is corrected for these contributions.

This is shown in Figure 3, where the $\mathrm{C}(1 \mathrm{~s})$ peak consists of two clearly separated contributions with differing chemical shifts: the lower binding energy contribution (around $295 \mathrm{eV}$, not corrected for sample charging), associated with the exclusively $\mathrm{C}-\mathrm{C}$ and $\mathrm{C}-\mathrm{H}$ bonded carbon, and the higher binding energy contribution (around $301 \mathrm{eV}$, not corrected for charging of the sample surface), associated with $C-F_{x}$ bonded carbon $(x>1)$. Since the sample charging is homogeneous (within the energy resolution of the analyzer), the relative positions of the different element signals can be compared, and from this comparison an "absolute" charging of around $9 \mathrm{eV}$ was determined. It is pointed out that the high binding energy

(33) Weber, J.; Janda, O.; Kavan, L.; Jegorov, A. J. Electroanal. Chem. $1986,199,81$

(34) (a) Handbook of X-ray photoelectron spectroscopy; Wagner, C. D., et al., Eds.; The Perkin-Elmer Corporation: Eden Prairie, MI, 1979. (b) Wagner, C. D.; Bickham, D. M. NIST Standard Reference Database 20, XPS Database, Version 1.0; US Department of Commerce, National Institute of Standards and Technology: Gaithersburg, MD, 1989.

(35) Scherer, G. C.; Pfluger, P. Proc. Electrochem. Soc. 1986, 86, 52.
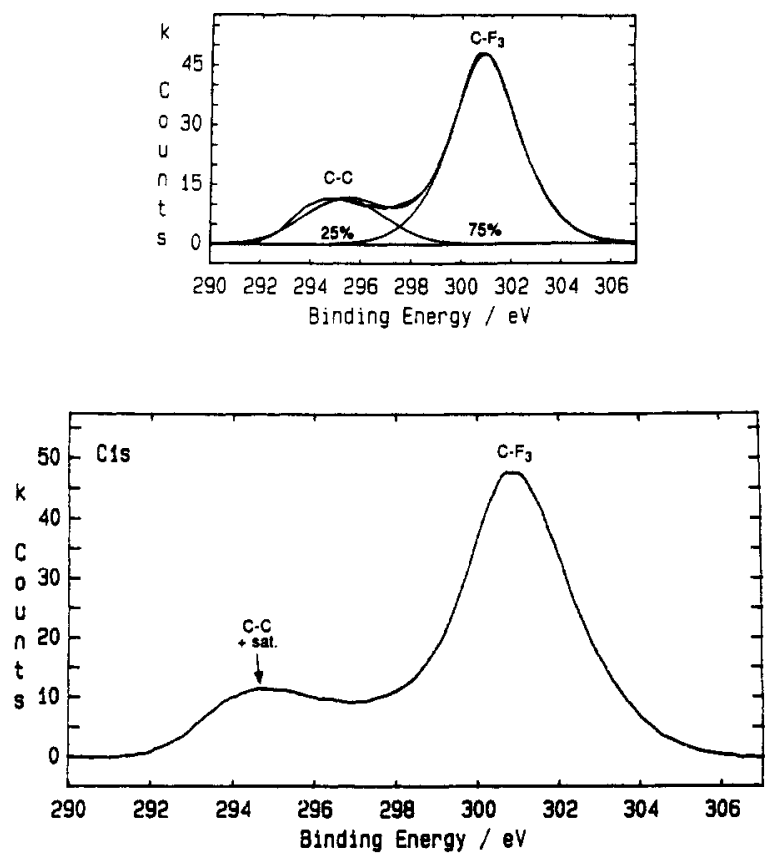

Figure 3. XPS carbon (C(1s)) spectrum of Nafion 117, showing contributions at binding energy around $295 \mathrm{eV}$ (not corrected for sample charging), associated with carbon atoms bonded only to carbon and/or hydrogen (represented as $\mathrm{C}-\mathrm{C}$ ), and around 301 $\mathrm{eV}$, associated with carbon atoms bonded as CFO- $\mathrm{CF}_{2}, \mathrm{CF}_{2}$ $\mathrm{CF}_{2}$, or $\mathrm{CF}_{3}$, (represented as $\mathrm{C}-\mathrm{F}_{3}$ ). Original spectrum also not corrected for background and $K \beta$ satellite (sat.) at around $8 \mathrm{eV}$ lower binding energy. Inset shows curve fitted spectrum, corrected for background and satellite, using two peaks associated with above mentioned carbon atoms, with relative contributions of $25 \%$ and $75 \%$ of total peak area.

"C-F ${ }_{x}$ " contribution actually consists of the superposition of the $\mathrm{CF}_{2}, \mathrm{CF}_{3}$, and CFO carbon chemical shifts, which are similar within experimental accuracy. The low binding energy " $\mathrm{C}-\mathrm{C} \& \mathrm{C}-\mathrm{H}$ " contribution in its turn consists of the superposition of the $\mathrm{Mg} \mathrm{K} \beta$ satellite (the contribution of which can be compensated for using software routines), possible hydrocarbon contamination, as well as degradation products. Since hydrocarbon contamination, notably associated with sample preparation and manipulation, can be neglected in the case of the Nafion membrane, ${ }^{27 a}$ and in view of the irradiation time-dependent increase of this contribution, it can be attributed to degradation of the membrane.

Using curve decomposition routines, the $C(18)$ signal can be decomposed quantitatively, yielding a degradation contribution within the analysis time of around $25-30 \%$ of the total carbon surface concentration (C(1s) peak area). If the surface carbon concentration is corrected for the degraded contribution, the $\mathrm{C} / \mathrm{F}$ ratio is in close agreement with the theoretical value, which is close to $1: 2$.

The $\mathrm{O}(1 \mathrm{~s})$ signal for pure Nafion is shown in Figure 4. Again, two contributions with different chemical shifts can be distinguished. In this case the contributions have a large overlap. The low binding energy contribution is, corrected for charging, located around $532 \mathrm{eV}$ and corresponds with that of the sulfonate groups in the membrane. ${ }^{34 a, b}$ The correction for charging is based on the $\mathrm{C}-\mathrm{C}$ contributions to $\mathrm{C}(1 \mathrm{~s})$ as a reference $(284.6 \mathrm{eV})$.

The higher energy contribution, shifted over $2 \mathrm{eV}$, is associated with the ether-type linkages in the polymer. These observations are corroborated with the $S(2 p)$ signal, which also shows a chemical shift attributed to sulfonatetype sulfur. The quantitative results, obtained from the $O(1 \mathrm{~s})$ curve decomposition and the surface sulfur concentration, respectively, are in agreement with the stoi- 




Figure 4. XPS $(O(18))$ spectrum of Nafion 117, showing contributions at binding energy of around $541 \mathrm{eV}$ (not corrected for sample charging) associated with oxygen atoms bonded as in sulfonate groups (represented as $\mathrm{SO}_{3}$ ) and around $544 \mathrm{eV}$ associated with oxygen atoms bonded in ether-type linkage to carbon (represented as C-O). This curve-fitted spectrum, using two peaks and corrected for background and satellite contributions shows fractions associated with each type of oxygen to be $35 \%$ and $65 \%$, respectively.
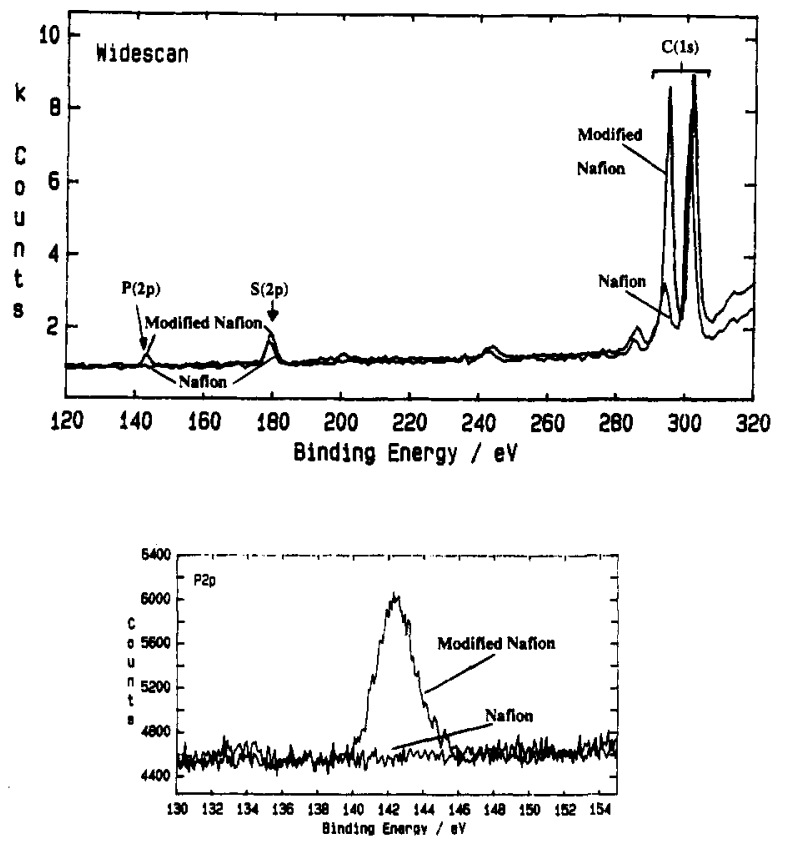

Figure 5. Superposition of widescan XPS spectra of Nafion 117 and modified Nafion 117 coated with $n$-heradecyltriphenylphosphonium amphiphiles (25 mM aqueous solution). Relevant signals, associated with $P, S$, and $C$ are indicated. Inset shows XPS P(2p) spectrum of Nafion 117 and modified Nafion 117 at binding energy of around $143 \mathrm{eV}$, showing clearly the presence of phosphorous atoms at the surface of the modified nafion.

chiometry for the sulfonate groups, i.e. $\mathrm{SO}_{3}$. These observations show that the sulfonate groups are present within the information depth of XPS, i.e. up to a few nanometers below the surface, which contrasts with the results reported by Kakuta et al ${ }^{36}$ on similar membranes. Also, it can be stated that, apart from irradiation-induced surface degradation which can be limited by using short acquisition times, the Nafion membrane surface resembles its bulk composition closely.

A similar conclusion is valid for the pure HTPB. $P$ and $\mathrm{Br}$ surface concentrations are the same within experimental accuracy, and the $\mathrm{P} / \mathrm{C}$ ratio reflects, again within experimental error, the bulk ratio. The observed oxygen is attributed to adsorbed water.

Figure 5 shows a XPS spectrum survey scan obtained from pure as well as HTP modified Nafion. No Br is observed at the surface of the HTP modified membrane, which indicates that the $\mathrm{Br}$ is substituted for another

(36) Kakuta, N.; White, J. M.; Campion, A.; Bard, A. J.; For, M. A.; Webber, S. E. J. Phys. Chem. 1985, 89, 48.
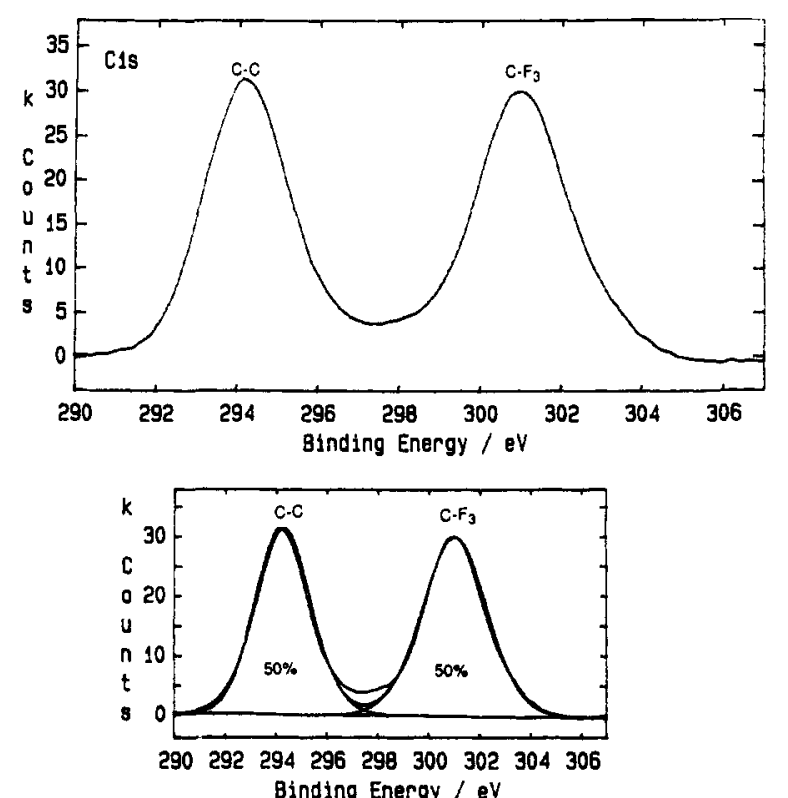

Figure 6. XPS C(1s) spectrum of Nafion 117 coated with $n$-hexadecyltriphenylphosphonium amphiphiles (25 mM aqueous solution). Contributions as described in Figure 1 are present now in differing proportions, around $50 \%$ each (see curve fit inset), due to presence at surface of amphiphile consisting (mainly) of carbon atoms bonded only to carbon and/or hydrogen. Same corrections valid as in Figure 3.

negatively charged moiety. These moieties are most probably the sulfonate groups, observed at the surface. It follows from Table I that the surface concentrations of $S$ and $P$ are of the same order of magnitude (1.2 and 0.7 atom \%, respectively), which is in agreement with this assumption. Assuming that HTP is mainly located at the surface of the membrane, and not in the bulk (see further), all amphiphile is therefore either present at the polymer interface, bonded to the surface sulfonate groups, or present as "free" HTPB. Since no Br is detected at the surface, it can be concluded that HTP is present in close contact to the sulfonate groups and forms a single layer at the polymer interface only. In order to analyze the possible effect of physisorption of HTPB to the surface, the amphiphile was applied to pure Teflon, which contains no polar (sulfonate) groups. Subsequent analysis with XPS showed no evidence for amphiphile at the surface of the Teflon, in agreement with the assumption of surface bonding of HTP to the sulfonate groups.

Figure 6 shows the C(18) peak shape of HTP modified Nafion. Clearly, the low binding energy $\mathrm{C}-\mathrm{C} / \mathrm{C}-\mathrm{H}$ contribution to the total carbon concentration has increased relative to the C(1s) peak of the pure Nafion (Figure 3). Surface areas below the XPS peak of a single element being directly proportional to surface concentrations, the modified Nafion shows roughly equal contributions of both carbons bound exclusively to carbon and hydrogen, and carbons of the type $-\mathrm{CF}_{2},-\mathrm{CFO}$, and $-\mathrm{CF}_{3}$. By use of curve fitting routines, the total carbon surface concentration associated with each type of moiety can be calculated. From Table I this is found to be 50\% of 46.7 atom \% for each type, or roughtly 23 atom \%. These concentrations can also be calculated starting from the bulk stoichiometry of both the membrane and the amphiphile (Table II), and using the XPS-determined surface concentrations of the "label" elements for Nafion (S) or HTP (P). This yields 1.2 atom $\% \times 20=24$ atom \% carbon and 0.7 atom $\% \times 34=24$ atom $\%$ carbon for the membrane and the amphiphile, respectively. These calculations, making use of known bulk stoichiometry, 

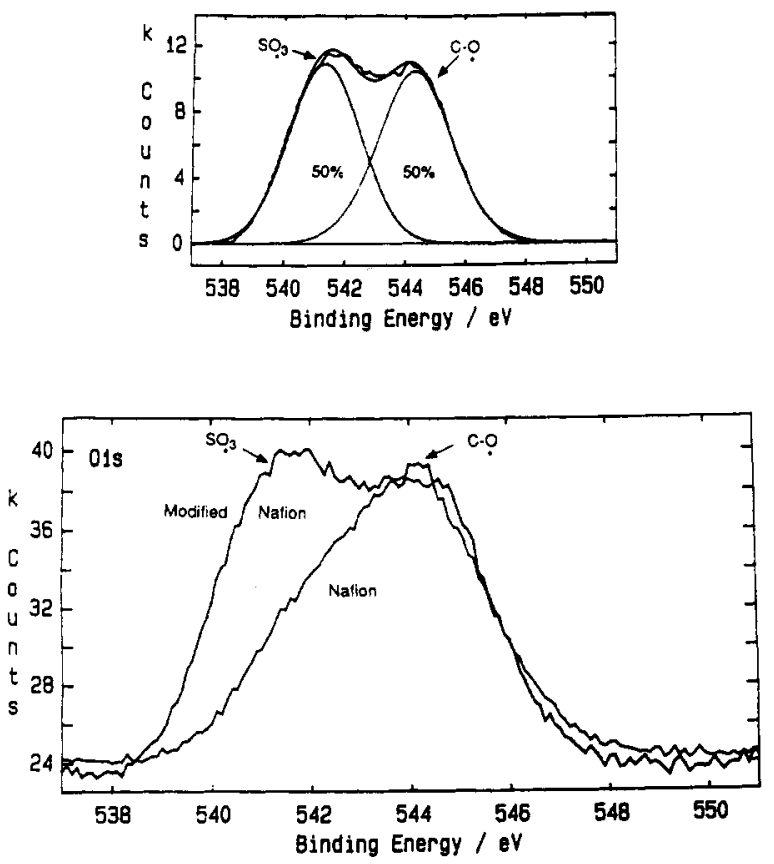

Figure 7. Superposition of XPS O(1B) spectra of Nafion 117 and Nafion 117 coated with $n$-hexadecyltriphenylphosphonium amphiphiles (25 mM aqueous solution), showing clearly higher low binding energy contribution in the latter, associated with the sulfonate-type oxygen. The curve fitted spectrum of modified Nafion (inset) shows around $50 \%$ for sulfonate-type, and $50 \%$ for ether-type oxygen, as compared to $35 \%$ vs $65 \%$, respectively, in Figure 4.

surface chemical state quantification by curve fitting, and element quantification by XPS, yield consistent results.

The observed P concentration for the HTP modified membrane is slightly lower than that of $\mathrm{S}$. This may indicate that not all the sulfonate groups are bonded to HTP. Alternatively, a fraction of the sulfonate groups may be shielded from the HTP (i.e. located just below the surface). On the other hand, the observed $S$ concentration for the HTP modified Nafion is higher than that of the pure Nafion surface. This indicates an enrichment of the polar groups at the surface of the membrane after modification. This indication is strongly supported by a comparison of the $\mathrm{O}(1 \mathrm{~s})$ signals of the pure and HTP modified Nafion. As shown in Figure 7 the latter shows a clearly higher low binding energy contribution associated with the sulfonate oxygen. The XPS results implicitly make use of the assumption of a surface region with homogeneous composition (via the empirically derived relative sensitivity factors). However, the observation of Br-free HTP, surface enrichment of sulfonate groups after amphiphile adsorbtion, and slightly lower HTP concentrations point to a model which consists of a homogeneously composed substrate with a thin amphiphile top layer presumed to be (less than) a monolayer.

It is expected that angle-resolved XPS (ARXPS) may yield evidence for the assumptions that HTP forms an adsorbed monolayer on a planar membrane surface. For example, if this model would be valid, it would lead to an increasing $F$ and decreasing $P$ concentration with increasing detection angle. The ARXPS results listed in Table I do not exhibit this trend. Consequently, both the homogeneous composition model used for the XPS quantification as well as the thin amphiphile layer on a flat substrate cannot be used to describe the observed phenomena. Although no macroscopic surface roughness (which may indeed influence the XPS detection) is assumed to be present, a topological model as that sche-

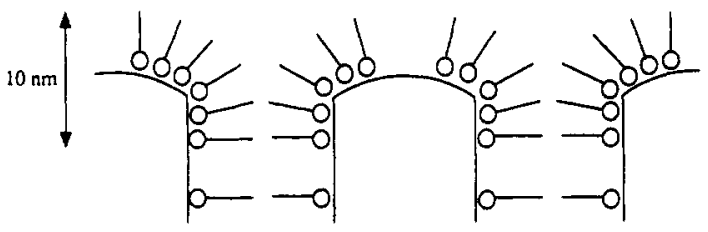

Figure 8. Model for the adsorption of $n$-hexadecyltriphenylphosphonium amphiphiles on a Nafion membrane based on XPS and SIMS experiments.


Figure 9. Positive ion static ToFSIMS spectrum of Nafion 117 coated with $n$-hexadecyltriphenylphosphonium amphiphiles (25 $\mathrm{mM}$ aqueous solution). Spectrum shows signals characteristic for Nafion (e.g. $31 \mathrm{~m} / e\left(\mathrm{CF}^{+}\right), 69 \mathrm{~m} / e\left(\mathrm{CF}_{3}{ }^{+}\right), 93 \mathrm{~m} / e\left(\mathrm{C}_{8} \mathrm{~F}_{3}{ }^{+}\right), \ldots$ ) as well as signals characteristic for the amphiphile (low mass "hydrocarbon peaks" and $261 \mathrm{~m} / \mathrm{e}$ and higher mass fragments, spaced every 14 amu, associated with alkyl triphenylphosphonium fragments; $487 \mathrm{~m} / \mathrm{e}$ i.e. amphiphile molecular ion).

matically drawn in Figure 8 is proposed. This model should further be validated with other techniques. To this end, static SIMS and X-ray induced energy dispersive $\mathrm{X}$-ray fluorescense analysis were applied. From this model, it follows that HTP may be present at the surface in regions with locally higher or lower concentrations (pores or domains). Also, the model would imply the presence of a certain amount of HTP in the bulk also (via the pores).

Static ToFSIMS experiments are in agreement with the XPS results. Static (i.e. quasi-nondestructive) SIMS is more surface sensitive than XPS (about $1 \mathrm{~nm}$ information depth), although it is only qualitative. Moreover, this technique provides laterally resolved information, the resolution being in the order of $1 \mu \mathrm{m}$. Analysis of HTPB shows the molecular ion peak $(487 \mathrm{~m} / \mathrm{e})$ in positive ion, and the $\mathrm{Br}$ isotopes in negative ion spectra. The pure Nafion shows the peaks characteristic for polytetrafluoroethylene, ${ }^{37}$ with additionally a peak at $80 \mathrm{~m} / e$ in the negative spectrum, which is associated with the sulfonate group.

The spectra obtained from the HTP modified Nafion are shown in Figures 9 and 10 for the positive and negative ion spectra, respectively. The amphiphile peaks are superimposed on the Nafion peaks. This indicates that

(37) Briggs, D.; Brown, A.; Vickerman, J. C. Handbook of static SIMS; J. Wiley and Sons: Chichester, 1989. 


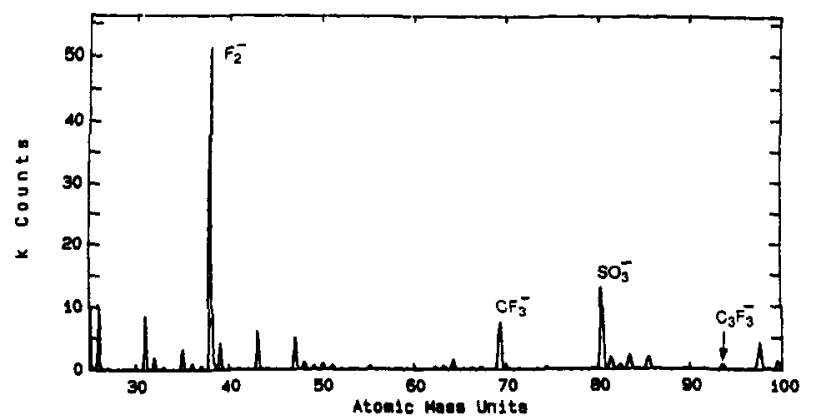

Figure 10. Negative ion static ToFSIMS spectrum of same compound as in Figure 9, showing characteristic peaks for Nafion (e.g. $38 \mathrm{~m} / \mathrm{e}\left(\mathrm{F}_{2}^{-}\right), 69 \mathrm{~m} / \mathrm{e}\left(\mathrm{CF}_{3^{-}}\right), 80 \mathrm{~m} / \mathrm{e}\left(\mathrm{SO}_{3}^{-}\right)$).

within the topmost $1 \mathrm{~nm}$, both amphiphile and membrane are present. Consequently, the coverage of the membrane by HTP is presumably less than a monolayer on the average. In agreement with the XPS results, the negative spectrum shows no $\mathrm{Br}$ peaks ( 79 and $81 \mathrm{~m} / \mathrm{e}$ ); only the $\mathrm{SO}_{3}$ peak at $80 \mathrm{~m} / \mathrm{e}$ is observed.

The possible inhomogeneous lateral distribution of sulfonate groups (and consequently the amphiphiles) over the surface was further investigated using static ToFSIMS imaging. By monitoring the distribution of the $80 \mathrm{~m} / \mathrm{e}$ (-ve ion) peak (i.e. the sulfonate-group characteristic signal) relative to the $38 \mathrm{~m} / e$ (-ve ion) peak associated with $\mathrm{F}_{2}$, i.e. characteristic for the membrane, it was not possible to see systematic intensity differences in the thus obtained parallel images, apart from those which can be attributed to topology phenomena (i.e. intensity differences the same in both images). This means that no lateral inhomogeneity in the amphiphile/sulfonate group distribution is present with characteristic dimensions larger than the resolution of the images, which is on the order of $1 \mu \mathrm{m}$. Obviously, the presence of smaller domain structures at the surface cannot be excluded from these experiments.

In order to investigate the presence of the amphiphile in a larger range below the surface, energy dispersive $X$-ray analysis of the membranes was performed. By use of X-ray rather than the conventional electron excitation, the degradation of the sample under the electron beam, as well as extensive sample preparation required to avoid heat and charge build-up, could be limited. The elementcharacteristic fluorescense $X$-rays could be observed using an energy dispersive detector. The information depth of this technique is in the range of several micrometers. This implies that the technique can be considered as a "bulk" analysis technique. The $\mathrm{C}, \mathrm{F}, \mathrm{S}$, and $\mathrm{P}$ signals of the modified Nafion indicate that P (as a "label" for HTP) is present in significantly lower concentrations than $S$ (as a "label" for Nafion). This indicates that the "bulk" HTP concentration measured in this way, is significantly smaller than the HTP concentration detected in the surface region using XPS. This is not conflicting with the adsorbtion and UV as well as ATR-IR experiments reported before, which indicate that a negligible amount of the HTP diffuses through the membrane.

\section{Conclusions}

From study of the adsorption of several positively charged amphiphiles on a Nafion membrane, it was found from conductivity measurements that $n$-alkyltriphenylphosphonium amphiphiles show the most stable adsorption behavior. UV spectroscopy showed that about $20 \%$ of the Nafion protons are exchanged for an amphiphile in case of $n$-hexadecyltriphenylphosphonium. No amphiphile is washed off upon replacing the amphiphile solution by water. No significant ion-exchange of $n$-hexadecyltriphenylphosphonium in deeper regions (below roughly $18 \mu \mathrm{m}$ ) of the Nafion membrane was detected from UV absorbtion experiments. By the absence of any bromide and the observation of a $S$ to $P$ ratio of nearly 1 (XPS), it was concluded that the outer sulfonate sites are ion-exchanged by the phosphonium amphiphile, forming a monolayer at the microscopic polymer interface. Inspection of the macroscopic interface to a depth of 1-10 nm by ATR-IR, XPS, and static SIMS measurements showed that $n$-hexadecyltriphenylphosphonium is present at the surface of the modified Nafion membrane. From angle-resolved XPS data (in the topmost 5-10 nm of the membrane) it is suggested that surface topology is responsible for the homogeneous distribution of the amphiphile over the membrane. Static time of flight SIMS data of the uppermost $1 \mathrm{~nm}$ layer indicate an incomplete coverage of the membrane by the amphiphile in agreement with XPS results. From bulk X-ray fluorescence measurements it follows that the amphiphile is present in the bulk also, although in significantly smallex concentrations than the surface concentration.

These results show that by adsorbing cationic amphiphiles on a Nafion membrane, thin amphiphilic (mono-)layers can be formed via an ionic bond with the sulfonate groups of the Nafion. Results from XPS and static SIMS analysis of the modified surface are consistent and show that these techniques are suited to analyze modified polymer surfaces. Work is in progress to immobilize selective macrocyclic carriers with cationic groups on the Nafion surface to obtain a thin and selective membrane. 